\title{
Full-Wave Modal Analysis of Slow-Wave Periodic Structures Loaded With Elliptical Waveguides
}

\author{
Stephan Marini, Ángela Coves, Member, IEEE, Vicente E. Boria, Senior Member, IEEE, and \\ Benito Gimeno, Member, IEEE
}

\begin{abstract}
In this paper, a novel full-wave method for the modal characterization of periodic structures loaded with elliptical waveguides is presented. This method relies on an integral equation formulation solved via the method of moments, which finally leads to the solution of a standard eigenvalue problem. The required modal spectrum of elliptical waveguides is determined through the boundary integral-resonant mode expansion technique. For validation purposes, the proposed analysis method is first successfully applied to periodic waveguide structures already considered in the technical literature. Then, our new algorithm is used to compute the related Brillouin diagrams and the interaction impedance of new periodic structures loaded with elliptical waveguides. Not only the main interacting mode (such as the $T M_{01}$ mode) is studied, but higher-order Floquet modes are also considered. These results have potential applications as slow-wave structures for high-power microwave devices and possibly filtering structures at millimeter-wave frequencies.
\end{abstract}

Index Terms-Arbitrarily shaped waveguides, Brillouin diagram, dispersion curves, electromagnetic band-gap, elliptical waveguide, Floquet modes, periodic structures.

\section{INTRODUCTION}

$\mathbf{M}$ ETALLIC periodic structures capable of supporting the propagation of slow electromagnetic waves are widely used for high power microwave device generation and for the acceleration of charged particles [1]. These slow-wave structures are designed to match the phase velocity of the propagating electromagnetic waves to the speed of electrons in the same structure to facilitate an effective beam/wave interaction [2]. It is important to know the dispersion relation for such devices to synchronize the phase velocity of the wave with an electron

Manuscript received August 26, 2009; revised November 4, 2009. This work was supported by the Ministerio de Educación y Ciencia, Spanish Government, under the coordinated Research Project TEC 2007/67630-C03-01. The review of this paper was arranged by Editor W. Menninger.

S. Marini is with the Departamento de Física, Ingeniería de Sistemas y Teoría de la Señal, Universidad de Alicante, 03690 Alicante, Spain (e-mail: smarini@dfists.ua.es).

Á. Coves is with the Departamento de Física y Arquitectura de Computadores, Universidad Miguel Hernández de Elche, 03202 Alicante, Spain (e-mail: angela.coves@umh.es).

V. E. Boria is with the Departamento de Comunicaciones, Instituto de Telecomunicaciones y Aplicaciones Multimedia, Universidad Politécnica de Valencia, 46022 Valencia, Spain (e-mail: vboria@ dcom.upv.es).

B. Gimeno is with the Departamento de Física Aplicada, Instituto de Ciencia de los Materiales, Universidad de Valencia, 46100 Valencia, Spain (e-mail: benito.gimeno@uv.es).

Color versions of one or more of the figures in this paper are available online at http://ieeexplore.ieee.org.

Digital Object Identifier 10.1109/TED.2009.2037175 beam [3]. In the last two decades, significant research effort has been directed to increasing the output power and operating frequency of the high-power Cerenkov-type microwave devices [4]. In most of the research and studies involving cylindrical slow-wave structures, it was assumed that the main interacting mode is the $T M_{01}$ symmetric mode [5], [6]. However, due to the asymmetries related to the electrons' distribution, as well as those attributed to manufacturing tolerances, higherorder modes can also be excited in the cited periodic structures [7]-[9]. In this context, it is important to examine the impact of higher-order modes and asymmetric modes on the dynamics of the electrons during the interaction process, particularly in systems operating at high frequency, where this problem may become critical [10]. Consequently, it is crucial to have an efficient and accurate numerical method for computing the dispersion relations of both symmetric and asymmetric modes in arbitrary slow-wave structures [7].

Charged particle beams of elongated elliptic cross sections have generated great interest in vacuum electronics due to their low space-charge energy and efficient coupling to RF structures as compared with circular beams [11]. Corrugated elliptical waveguides used as slow-wave structures provide a series of advantages: high power capacity, good thermal conductivity, large size, and high precision of manufacturing and assembling [12]. The corrugated elliptical waveguide was studied in [13] with an approximate analysis, which is valid for small values of eccentricity, using only a single term of Mathieu function series. Raevskii and Smorgonskii and Belov et al. studied the structure characteristics as a microwave transmission line [14], [15]. Xu et al. studied, using a field-matching method, the fundamental mode of the corrugated elliptical waveguide by taking no account of space harmonics, as well as neglecting higher-order modes in the slot region [16], [17]. Recently, Han et al. have studied [18], by following a modematching approach, the dispersion characteristics of three even TM modes, considering space harmonics in the inner region and higher-order modes in the slot region. Nevertheless, all the methods considered are based on the computation of Mathieu functions, which cannot be very efficient in terms of computational cost. In this paper, a full-wave modal analysis of slow-wave periodic structures loaded with elliptical waveguides is presented. To solve the proposed elliptical geometries, we will make use of a rigorous and efficient method based on the integral equation analysis technique described in [19]. The application of this technique requires knowing the modal chart of all arbitrarily shaped waveguide sections involved in 


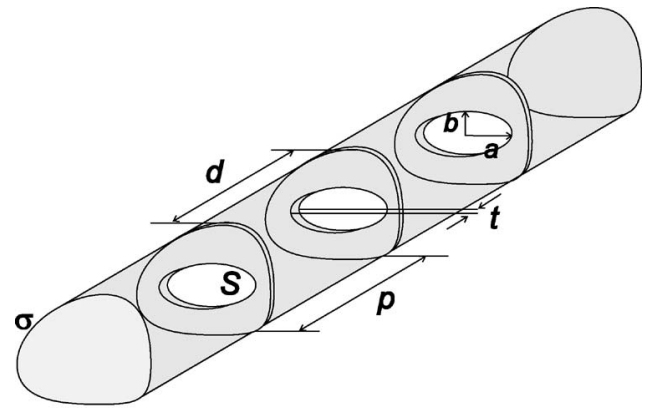

(a)

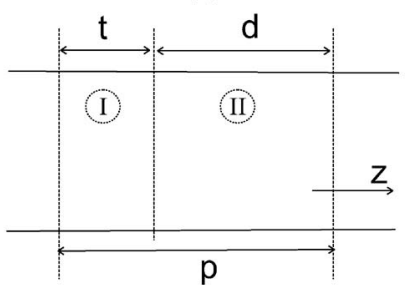

(b)

Fig. 1. (a) Periodic structure loaded with elliptical waveguides. (b) Side view of the unit cell.

the periodic structure under consideration. For this purpose, the well-known boundary integral-resonant mode expansion (BI-RME) method, originally proposed in [20] and recently revisited in [21], is employed.

After solving the waveguide steps and the uniform sections involved in a single cell of the periodic structure, a standard eigenvalue problem is formulated in terms of the transmission $(A B C D)$ chain matrix, whose solution provides the propagation constants of the fundamental and all higher-order Floquet modes (both symmetric and asymmetric). A method based on the correlation between eigenvectors at two different frequencies is used to trace the dispersion diagram, thus allowing to identify multimode regions and the proper sorting of supported propagating and evanescent modes. The interaction impedance between the RF wave and the electron beam for different periodic structures is also successfully computed.

To fully validate the accuracy of the method proposed in this paper, we have first applied it to analyze different periodic structures already considered in previous publications, including not only the fundamental mode but also higherorder modes. Then, with our novel characterization method, we have studied the effects, in the $k_{0}-\beta$ diagram, of two nonconfocal cases shifting the elliptical waveguide iris and producing an asymmetry of the structure. Finally, the Brillouin diagram considering the first five Floquet propagating modes (such as $T M / T E_{2 m n}$ ) and the mean coupling impedance of a new periodic circular structure loaded with elliptical irises are computed and compared with the circular irises case. Note that the time factor $e^{j \omega t}$ is considered and omitted throughout this paper.

\section{THEORY}

\section{A. Dispersion Equation}

In this section, we will present the theoretical basics related to the proposed analysis technique. As shown in Fig. 1(a), the periodic structure under consideration consists of the cascade connection of an arbitrarily shaped waveguide, whose cross section $\sigma$ is defined by any combination of linear, circular, and/or elliptical arcs, and an elliptical waveguide iris. The unit cell of length $p$ consists of two regions [see Fig. 1(b)], where $d$ represents the length of the groove region, and $t=p-d$ represents the thickness of the elliptical iris.

The $A B C D$ chain matrix of one period $p$ of the structure under study $(\mathbf{M})$ is directly obtained by solving the cascade connection of two waveguides and related steps as

$$
\mathbf{M}=\left[\begin{array}{ll}
\mathbf{A} & \mathbf{B} \\
\mathbf{C} & \mathbf{D}
\end{array}\right]=\prod_{i=1}^{4}\left[\begin{array}{ll}
\mathbf{A}_{\mathbf{i}} & \mathbf{B}_{\mathbf{i}} \\
\mathbf{C}_{\mathbf{i}} & \mathbf{D}_{\mathbf{i}}
\end{array}\right]
$$

where the coefficients of the first and third $A B C D$ matrices are trivial, since they correspond to hollow waveguides with finite lengths. The second and fourth matrices are related to the modeling of the same planar waveguide junction.

To obtain the full-wave characterization of the involved planar junction, a very efficient method based on the numerical solution of a first-kind Fredholm integral equation, originally described in [19] for dealing with rectangular waveguides, has properly been updated. The objective of this technique is to obtain a multimodal representation of each planar waveguide junction in terms of a generalized impedance matrix. A remarkable contribution of this method is the distinction made between accessible and localized modes: accessible modes are those used to connect transitions, whereas localized modes are only used to describe the electromagnetic fields in the junction (the number of localized modes is always greater than the number of accessible ones).

The application of this efficient full-wave analysis method requires knowledge of the modal charts related to the waveguides of the planar junction under consideration, as well as the coupling coefficients between the modes of these waveguides. To obtain this information, the well-known BI-RME technique, which was originally proposed in [20], is employed. More details about the extension of such technique for the rigorous consideration of arbitrary cross sections, defined by any combination of linear, circular and/or elliptical segments, can be found in [21].

Once the $2 \times 2$ block generalized $Z$ matrix of the planar waveguide junction is determined, the corresponding transmission $(A B C D)$ parameters are derived as follows:

$$
\begin{aligned}
& \mathbf{A}=\mathbf{Z}_{11} \cdot \mathbf{Z}_{21}^{-1} \\
& \mathbf{B}=\mathbf{Z}_{11} \cdot \mathbf{Z}_{21}^{-1} \cdot \mathbf{Z}_{22}-\mathbf{Z}_{12} \\
& \mathbf{C}=\mathbf{Z}_{21}^{-1} \\
& \mathbf{D}=\mathbf{Z}_{21}^{-1} \cdot \mathbf{Z}_{22}
\end{aligned}
$$

and then the transmission matrix $\mathbf{M}$ can easily be computed using (1). We must now remind that in a periodic structure of period $p$, a generic component $F(z)$ of the electromagnetic field must satisfy the Floquet condition [22]

$$
F(z+p)=e^{-\gamma p} F(z) \quad \forall z
$$


where $\gamma$ is the propagation constant of the modes of the infinite periodic structure. Imposing the Floquet condition at the unit cell, we obtain

$$
\left(\begin{array}{c}
\mathbf{V}_{1} \\
\mathbf{I}_{1}
\end{array}\right)=\mathbf{M}\left(\begin{array}{c}
\mathbf{V}_{2} \\
-\mathbf{I}_{2}
\end{array}\right)=e^{\gamma p}\left(\begin{array}{c}
\mathbf{V}_{2} \\
-\mathbf{I}_{2}
\end{array}\right)
$$

The right-hand side of (7) can be expressed in classical canonical form

$$
\mathbf{M} \cdot \mathbf{x}=\mathbf{\Lambda} \cdot \mathbf{I} \cdot \mathbf{x}
$$

where $\mathbf{I}$ is the identity matrix. The solutions ( $\boldsymbol{\Lambda}$ and $\mathbf{x})$ of this standard eigenvalue problem, which are related, respectively, to the required propagation constants (eigenvalues) and the transverse field distributions (eigenvectors) in the periodic structure, can straightforwardly be determined using well-established routines.

At a given frequency, the real and imaginary parts of the propagation constant, i.e., $\gamma=\alpha+j \beta$, corresponding to a Floquet mode are directly related to the eigenvalue $\Lambda$ through the following relationships:

$$
\alpha=\frac{\ln |\Lambda|}{p} \quad \beta=\frac{\angle \Lambda}{p} .
$$

We must underline that the number of eigenvalue solutions $\Lambda$ in the canonical problem defined by (8) is equal to the order of the matrix $\mathbf{M}$ in the left-hand side. These eigenvalues are, in general, complex, appearing as pairs corresponding to forward and backward directions of propagation of the same mode. Passbands and stopbands are characterized, respectively, by the condition as to whether the periodic structure supports at least one propagating Floquet mode within the frequency range considered.

In conclusion, the nontrivial solutions of the problem and the resulting points of the dispersion diagram are solved frequency by frequency. It may happen that, for instance, when several modes are simultaneously excited (multimode region), the solutions are mixed up since the eigenvalues at different frequencies do not appear in the same order. To overcome this problem, we have followed a method originally proposed in [23] based on computing the correlation between eigenvectors at two different frequency points.

This correlation-based method computes, for any subinterval $\left[f_{1}, f_{2}\right]$ of the frequency band under analysis, the eigenvalue vectors $\boldsymbol{\Lambda}_{\mathbf{1}}$ and $\boldsymbol{\Lambda}_{\mathbf{2}}$ and the corresponding eigenvector matrices $\mathbf{V}_{\mathbf{1}}$ and $\mathbf{V}_{\mathbf{2}}$ at $f_{1}$ and $f_{2}$, respectively. The eigenvalues have to be arranged in ascending order of amplitude, and the eigenvectors are orthogonal and normalized according to

$$
\mathbf{V}_{1}^{\mathbf{T}} \mathbf{V}_{1}^{*}=\mathbf{I} \quad \mathbf{V}_{2}^{\mathbf{T}} \mathbf{V}_{2}^{*}=\mathbf{I}
$$

where $\mathrm{T}$ denotes the transpose matrix, and $*$ denotes the complex conjugate.

To find the correct sorting of the solutions, a correspondence between the eigenvalue vectors $\Lambda_{1}$ and $\Lambda_{2}$ is needed. This is obtained by calculating a correlation matrix between the corresponding eigenvectors as

$$
\mathbf{R}=\mathbf{V}_{1}^{\mathbf{T}} \mathbf{V}_{2}^{*}
$$

Due to the normalization condition (10), if the $i$ th eigenvector at frequency $f_{1}$ slightly differs from the $j$ th eigenvector at frequency $f_{2}$, the element $R_{i j}$ is close to unity. Therefore, there is a strong correlation between $\Lambda_{1 i}$ and $\Lambda_{2 j}$. Conversely, $R_{i j}$ is close to zero when there is no correlation between the two involved eigenvalues. It is important to note that the eigenvalues are ordered, so the correlation matrix $\mathbf{R}$ usually exhibits unity elements in the main diagonal. If the position of two corresponding eigenvalues are different at $f_{1}$ and $f_{2}$, then the matrix $\mathbf{R}$ exhibits elements close to unity out of the main diagonal. A threshold procedure is applied to $R_{i j}$ elements to obtain a new matrix where in any row or column only appears one element equal to unity, and all the other elements are zero. This procedure detects crossings between eigenvalues and therefore allows, in a fully automated way, the correct computation of the dispersion curve in a frequency range considering all interactions among the higher-order modes.

Finally, we must say that the computational effort related to the whole procedure described for finding the dispersion diagram of periodic waveguide structures is rather low. On one hand, the canonical eigenvalue problem outlined in (8) is quickly solved, since the dimension of the $\mathbf{M}$ matrix (directly related to the number of accessible modes chosen in the waveguides) is typically small. On the other hand, the modal analysis of arbitrarily shaped waveguides is frequency independent, so it is performed only once and outside the frequency loop.

\section{B. Coupling Impedance and $E_{z}$ Field Computation}

The coupling impedance is an important parameter in a linear-beam tube, since it is a measure of the interaction between an RF wave and the electron beam [17]. The mean coupling impedance of the $n$th Floquet mode (TM family) is defined as

$$
K_{c}=\frac{\overline{E_{z_{n}} E_{z_{n}}^{*}}}{2 \beta_{n}^{2} P}
$$

where $\overline{E_{z_{n}}}$ is the average longitudinal component of the electric field on the $n$th Floquet mode over the cross section of the junction (the elliptical iris section in this case), and $\overline{E_{z_{n}}^{*}}$ is its conjugate value; $\beta_{n}$ is the imaginary part of the $n$th Floquet mode propagation constant, and $P$ is the total power flow through the whole structure (related to the Poynting vector). Therefore, we have

$$
\overline{E_{z_{n}} E_{z_{n}}^{*}}=\frac{1}{S} \int_{S}\left|E_{z_{n}}\right|^{2} d S
$$

where $S$ is the elliptical iris section, and

$$
P=\frac{1}{2} \sum_{i} \operatorname{Re} \int_{S} \mathbf{E}_{\mathbf{i}} \times \mathbf{H}_{\mathbf{i}}^{*} \cdot \widehat{z} d S
$$


with $\mathbf{E}_{\mathbf{i}}$ and $\mathbf{H}_{\mathbf{i}}$ being the vector modal fields of the elliptical iris waveguide.

To evaluate (12), it is required to know the complete modal chart of the involved elliptical waveguides in a very accurate way. To reach this aim, the well-known BI-RME method (see [20] and [21]) is proposed to be used. Following this method, the longitudinal electric field is calculated using

$$
\begin{aligned}
e_{z}(\mathbf{r}) & =\frac{k_{c}^{2}}{k}\left[\int_{\sigma} g\left(\mathbf{r}, \mathbf{s}^{\prime}\right) J_{z}\left(l^{\prime}\right) d l^{\prime}+\sum_{m} \frac{\psi_{m}(\mathbf{r})}{k_{m}^{\prime 2}} a_{m}^{\prime}\right] \\
a_{m}^{\prime} & =\frac{k^{2}}{k_{m}^{\prime 2}-k^{2}} \int_{\sigma} \psi_{m}\left(\mathbf{s}^{\prime}\right) J_{z}\left(l^{\prime}\right) d l^{\prime}
\end{aligned}
$$

where $\mathbf{r}$ and $\mathbf{s}^{\prime}$ are a generic observation and a source point, respectively; $k=\omega \sqrt{\mu \epsilon}$ is the wavenumber; $g$ is the static 2-D Green's function (satisfying Dirichlet's boundary condition) for a rectangular resonator that involves the arbitrary waveguide; $k_{m}^{\prime}$ 's are the cutoff wavenumbers for the TM modes of such rectangular waveguide enclosure, and $\psi_{m}(\mathbf{r})$ is the corresponding normalized scalar potentials; $J_{z}\left(l^{\prime}\right)$ is the longitudinal surface current density on $\sigma$; and $k_{c}$ and $a_{m}^{\prime}$ are the cutoff wavenumbers for the calculated arbitrary waveguide and the modal expansion coefficients, respectively. The last two unknown parameters are obtained after the expansion of the surface current density in terms of piecewise parabolic functions

$$
J_{z}\left(l^{\prime}\right)=\sum_{j} b_{j}^{\prime} u_{j}\left(l^{\prime}\right)
$$

and solving an integral equation via the Galerkin version of the method of moments, where the basis and testing functions $u_{j}$ are chosen to be overlapping piecewise parabolic splines. Finally, to obtain the complete field expression of the $n$th Floquet mode, it is necessary to add the wave propagation in the $z$ direction and the modal amplitudes

$$
E_{z_{n}}=-j \eta \sum_{i} I_{i}^{(n)} e_{z_{i}} e^{-j \widetilde{\beta_{i}} z}
$$

where $I_{i}^{(n)}$, obtained from (7), is the eigenvector corresponding to the $n$th Floquet mode, $\eta=\sqrt{\mu / \epsilon}$ is the characteristic wave impedance, and $\widetilde{\beta}_{i}$ is the propagation constant of the $i$ th arbitrarily shaped waveguide mode. Now, the Poynting vector can easily be obtained because the electric $\mathbf{e}_{\mathbf{m}}$ and magnetic $\mathbf{h}_{\mathbf{m}}$ waveguide modes have been normalized according to

$$
\int_{S} \mathbf{e}_{\mathbf{m}} \times \mathbf{h}_{\mathbf{m}}^{*} \cdot \widehat{z} d S=1
$$

so we obtain

$$
P=\frac{1}{2} \sum_{i} R e\left(V_{i} I_{i}^{*}\right)
$$

where $V_{i}$ and $I_{i}$ are the equivalent voltage and current of the $i$ th mode of the arbitrarily shaped waveguide (elliptical iris in this case).

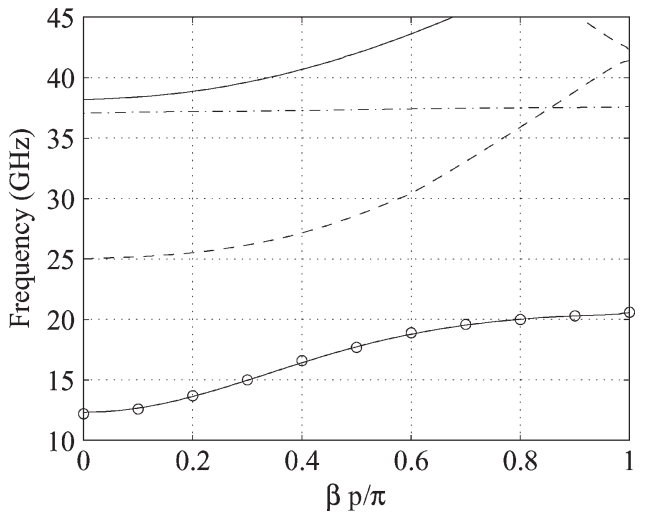

Fig. 2. Brillouin diagram for a periodic elliptical structure with major and minor semi-axis dimensions $a_{1}=8.62 \mathrm{~mm}, b_{1}=7.02 \mathrm{~mm}, a_{2}=11.19 \mathrm{~mm}$, $b_{2}=10.02 \mathrm{~mm}$, respectively; $p=4 \mathrm{~mm}$; and $d=2 \mathrm{~mm}$. The solid line corresponds to the first symmetric Floquet mode, the dashed line to the second symmetric Floquet mode, and the dash-dotted line to the third symmetric Floquet mode. The circles are taken from [16] for comparison.

\section{RESULTS}

Next, we proceed to study the accuracy and efficiency of the proposed analysis technique through several examples. First, we have computed and compared the dispersion diagram and the interaction impedance of elliptical corrugated waveguides presented in [16] and [18]. Second, with our novel characterization method, we have studied the effects, in the $k_{0}-\beta$ diagram, of two nonconfocal periodic elliptical waveguides by shifting the elliptical iris in the $x$ - and $y$-axes, respectively. A misalignment of the elliptical irises caused by manufacturing tolerances can produce an asymmetry of the whole structure and consequently the appearance of asymmetric modes. Finally, a new periodic circular structure loaded with elliptical waveguide irises is considered. The Brillouin diagram and the mean coupling impedance are presented and compared with the circular iris case. Higher-order Floquet modes are also computed and plotted. All the reported computational efforts have been obtained with an Intel Core 2 platform at $2.4 \mathrm{GHz}$ with 1-GB RAM.

\section{A. Corrugated Elliptical Waveguides}

First of all, to validate our theory and the developed computer code, we have considered the corrugated elliptical waveguide structures studied in [16]. In this paper, the authors use space harmonic effects and the field matching method to compute the field components, ignoring higher-order modes in the slot region. Therefore, all the results presented in [16] are refereed to the fundamental lowest symmetric ( ${ }_{e} T M_{01}$ for the elliptical case or $T M_{01}$ for the circular one) mode. Fig. 2 shows the Brillouin diagram of a periodic elliptical structure (i.e., a stepwise cascade connection of two different elliptical waveguides), with dimensions $a_{1}=8.62 \mathrm{~mm}, b_{1}=7.02 \mathrm{~mm}, a_{2}=11.19 \mathrm{~mm}$, $b_{2}=10.02 \mathrm{~mm}, p=4 \mathrm{~mm}, d=2 \mathrm{~mm}$, and $a_{i}$ and $b_{i}$ being the major and minor semi-axis of the elliptical waveguides, respectively. The solid line corresponds to the first symmetric Floquet mode, the dashed line to the second symmetric Floquet mode, and the dash-dotted line to the third symmetric Floquet mode, which have clearly been distinguished by our analysis 


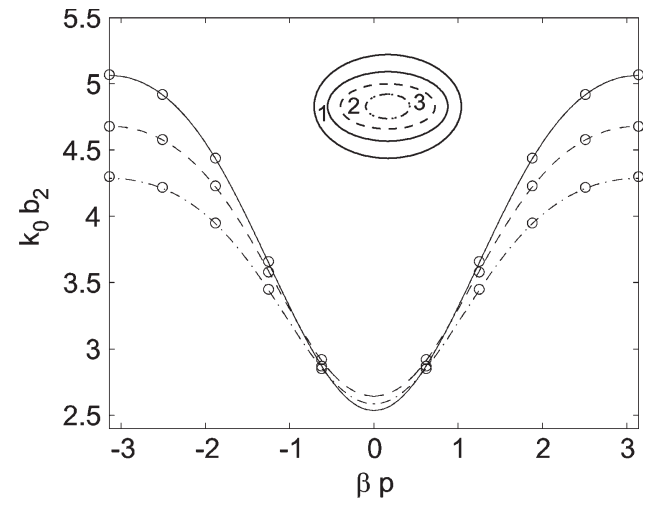

Fig. 3. $k_{0}-\beta$ diagram for periodic elliptical structures with different values of eccentricity $e_{1}$ while the eccentricity of the groove elliptical waveguides $e_{2}$ is constant. The dimensions for the bigger elliptical waveguides are $a_{2}=$ $5.596 \mathrm{~mm}, b_{2}=5.007 \mathrm{~mm}, p=2 \mathrm{~mm}$, and $d=1 \mathrm{~mm}$. The solid line corresponds to the case $a_{1}=4.599 \mathrm{~mm}$ and $e_{1}=0.5436$; the dashed line to $a_{1}=4.4523 \mathrm{~mm}$ and $e_{1}=0.5615$; and finally the dash-dotted line to $a_{1}=$ $4.3081 \mathrm{~mm}$ and $e_{1}=0.5803$. The circles are taken from [16] for comparison.

technique. Very good agreement between our results and those reported in [16] for the fundamental mode can be observed.

In Fig. 3 , we present the variations of the $k_{0}-\beta$ diagram of the fundamental mode with three different values for the eccentricity $e_{1}$ of the elliptical iris, which is defined as $e_{i}=$ $\sqrt{a_{i}^{2}-b_{i}^{2}} / a_{i}$, whereas the groove elliptical waveguide dimensions are constant $\left(e_{2}=0.4466\right)$. The elliptical corrugated structure has a period of $p=2 \mathrm{~mm}$, and the length of the groove region is $d=1 \mathrm{~mm}$. The elliptical iris eccentricity is taken as $e_{1}=0.5436$ for the first case, $e_{1}=0.5615$ for the second case, and $e_{1}=0.5803$ for the third case, respectively. The dimensions for all the waveguides are included in the caption of Fig. 3. From this figure, we can conclude that our results are in excellent agreement with those obtained from [16] (circles) for the fundamental lowest symmetric mode. As described by the authors in [16], a gradual increase in the eccentricity $e_{1}$ decreases the size of the interaction region, forcing the structure to approach a coupled cavity chain; thus, increasing the eccentricity $e_{1}$ can increase the dispersion of the corrugated elliptical waveguide while $e_{2}$ is a constant.

The next example compares the corrugated elliptical waveguide structure with the corrugated circular case with bigger radius $a=5.007 \mathrm{~mm}$ and inner iris radius $b=3.5085 \mathrm{~mm}$. Two kinds of corrugated elliptical waveguide structures are considered. In the first kind, the sections of the elliptical waveguide are inscribed ellipses of the circular waveguides $\left(a_{1}=3.5085 \mathrm{~mm}\right.$, $b_{1}=2.8805 \mathrm{~mm}, a_{2}=5.007 \mathrm{~mm}, b_{2}=4.591 \mathrm{~mm}$, i.e., major axis length constant with regard to the circular case). In the second kind, the sections of the elliptical waveguide are circumscribed ellipses of the circular waveguides $\left(a_{1}=4.308 \mathrm{~mm}\right.$, $b_{1}=3.5085 \mathrm{~mm}, a_{2}=5.596 \mathrm{~mm}, b_{2}=5.007 \mathrm{~mm}$, i.e., minor axis length constant with respect to the circular case). In Fig. 4, the results predicted by our method are successfully compared with those found in [16] (represented with circles). From the $k_{0}-\beta$ diagram, it can be seen that for the inscribed ellipses case, the dispersion of the structure will slightly strengthen in comparison with the circular structure. If the sections of the elliptical waveguides are circumscribed ellipses in the circular

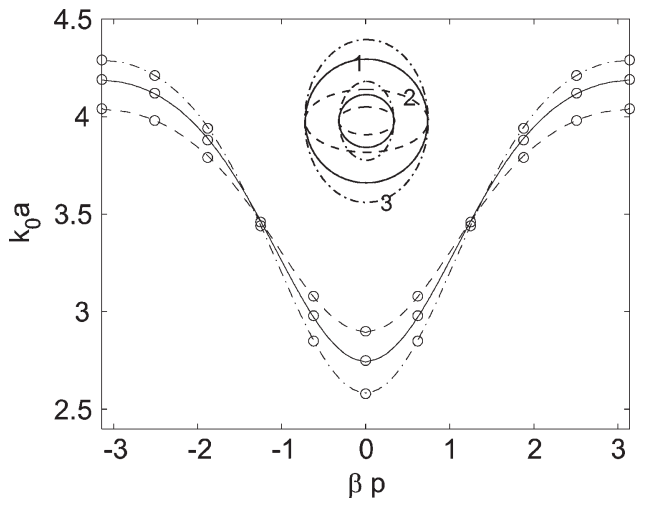

Fig. 4. $k_{0}-\beta$ diagram for three different periodic structures. The solid line shows the dispersion curve for the circular periodic case with bigger radius $a=$ $5.007 \mathrm{~mm}$ and inner radius $b=3.5085 \mathrm{~mm}$. The dashed line corresponds to the periodic elliptical structure with $a_{1}=3.5085 \mathrm{~mm}, b_{1}=2.8805 \mathrm{~mm}, a_{2}=$ $5.007 \mathrm{~mm}$, and $b_{2}=4.591 \mathrm{~mm}$ (inscribed ellipses of the circular waveguide); and in dash-dotted line, a periodic elliptical structure with $a_{1}=4.308 \mathrm{~mm}$, $b_{1}=3.5085 \mathrm{~mm}, a_{2}=5.596 \mathrm{~mm}$, and $b_{2}=5.007 \mathrm{~mm}$ (circumscribed ellipses of the circular case). For all the cases, $p=2 \mathrm{~mm}$, and $d=1 \mathrm{~mm}$. The circles are taken from [16] for comparison.

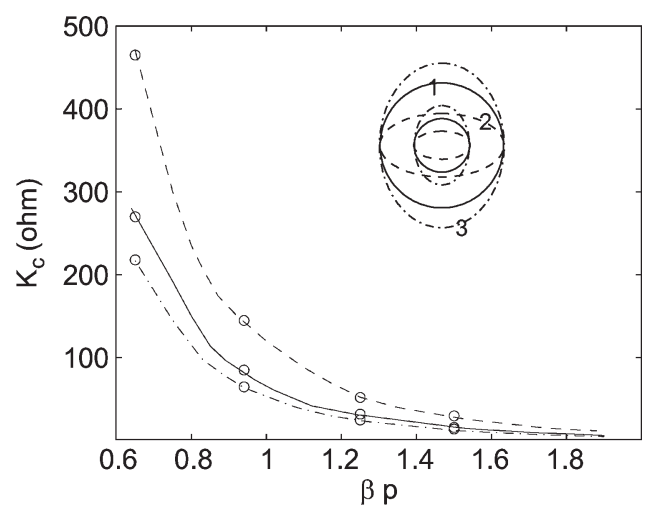

Fig. 5. Mean coupling impedance for the same periodic structures in Fig. 4. (Solid line) Circular case. (Dashed line) Inscribed periodic elliptical case. (Dash-dotted line) Circumscribed periodic elliptical case. The circles are taken from [16] for comparison.

waveguides, the dispersion will weaken, and the passband bandwidth increases.

Fig. 5 shows the interaction impedance for the three structures considered before computing with our method and the results presented in [16]. As can be observed, an excellent agreement between both results is obtained up to $\beta p=1.7$; for high frequencies, the coupling impedance decreases very slowly. From these results, we can remark that decreasing the minor axis length increases the coupling impedance. This occurs because the axial component of the electric field becomes stronger, decreasing $b_{1}$ [16]. Increasing the length of the major axis decreases the coupling impedance. Therefore, if the sections of the elliptical waveguide are inscribed ellipses of the circular waveguides, we can obtain an increase of the dispersion behavior and the interaction impedance compared with the circular one.

Once the new proposed theory has successfully been validated, computing the dispersion characteristic of the lowest symmetric mode, we have performed a further verification considering higher-order symmetric modes. In the next example 


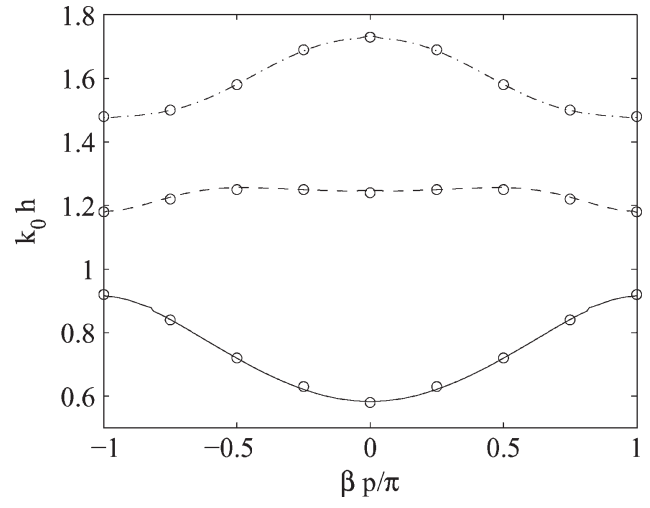

Fig. 6. Dispersion curves for corrugated elliptical waveguide with dimensions $a_{1}=6.772 \mathrm{~mm}, b_{1}=6.528 \mathrm{~mm}, a_{2}=8.222 \mathrm{~mm}, b_{2}=8.022 \mathrm{~mm}, p=$ $6 \mathrm{~mm}$, and $d=3 \mathrm{~mm}$. The constant $h=e a=1.8 \mathrm{~mm}$ represents half of the distance between foci. (Solid line) First symmetric Floquet mode. (Dashed line) Second symmetric Floquet mode. (Dash-dotted line) Third symmetric Floquet mode. The circles are taken from [18] for comparison.

(see Fig. 6), we have calculated the dispersion curves of the first three symmetric modes for a corrugated elliptical waveguide (i.e., the first three ${ }_{e} T M_{0 n}$ modes). The circles are taken from [18] considering higher-order modes in the slot region; good agreement is observed in all curves. Passbands and stopbands between adjacent modes are correctly identified. All the previous results were obtained using 150 accessible modes, 200 basis functions, and 400 kernel terms (i.e., localized modes [19]) for solving the planar waveguide junction of the structure. To compute the displayed dispersion diagram, the first four $e_{e} T M_{n m}$ symmetric modes have been used for solving (8). The complete analysis procedure has required a global computational effort of $0.69 \mathrm{~s}$ per frequency point. Such numerical effort is very well compared with the ones required by available commercial software tools than can cope with this kind of elliptical structure.

\section{B. New Periodic Structures Loaded With Elliptical Waveguides}

Next, we will consider new periodic structures loaded with elliptical waveguides. For instance, in the first two examples, we have studied the Brillouin diagram of periodic corrugated elliptical waveguides when the elliptical waveguide irises are shifted in the $x$ - and $y$-axes, respectively, in comparison with the confocal case. The geometry and the dimensions of the three structures analyzed are presented in Fig. 7. A misalignment of the elliptical irises, attributed to manufacturing tolerances, produces an asymmetry of the whole structure, and consequently, asymmetric modes can appear. Our method allows to analyze the structure considering the effects of all these modes. Fig. 7 compares the $k_{0}-\beta$ diagram of the lowest symmetric Floquet mode for a confocal case (solid line) and for two nonconfocal cases (in dashed 0.2-mm $x$-axis shifted and in dash-dotted 0.5 -mm $x$-axis shifted). As can be seen, an increase of the $x$-axis misalignment produces a decrease of the passband bandwidth; meanwhile, the dispersion characteristics change slightly. However, if the misalignment is in the $y$-axis, Fig. 8 shows a strength increase in the bandwidth and a decrease of the dispersion. This decrease is strengthened when increasing the misalignment in the $y$-axis (see the dash-dotted line in Fig. 8),

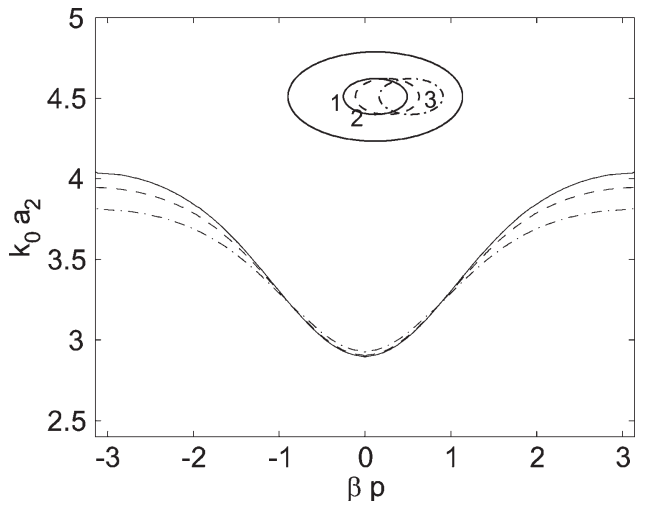

Fig. 7. $k_{0}-\beta$ diagram of the lowest symmetric Floquet mode for three different periodic elliptical structures: a confocal case (solid line) and two nonconfocal cases (0.2- and 0.5-mm $x$-axis shifted, in dashed and dash-dotted lines, respectively). The dimensions are $a_{1}=3.508 \mathrm{~mm}, b_{1}=2.88 \mathrm{~mm}, a_{2}=$ $5.007 \mathrm{~mm}, b_{2}=4.591 \mathrm{~mm}, p=2 \mathrm{~mm}$, and $d=1 \mathrm{~mm}$.

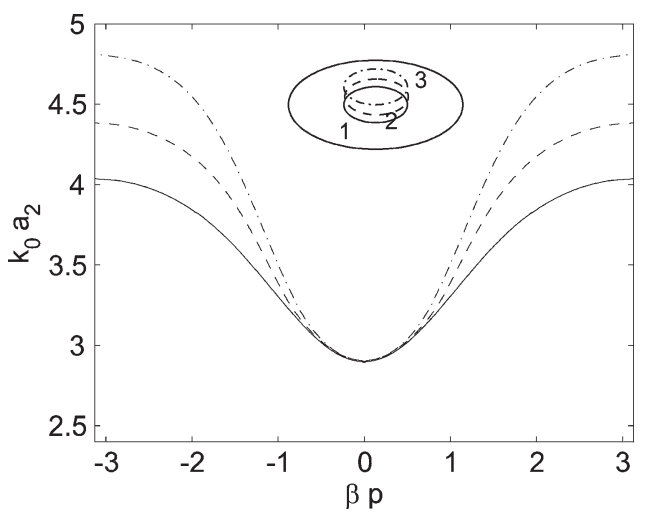

Fig. 8. $k_{0}-\beta$ diagram for three periodic elliptical structures: a confocal case (solid line) and two nonconfocal cases $(0.2-$ and 0.5 - $\mathrm{mm} y$-axis shifted in dashed and dash-dotted line, respectively). The dimensions are described in Fig. 7.

since the fields for the fundamental mode are varying more quickly in this vertical direction.

The last example considered in this paper deals with a periodic circular waveguide loaded with elliptical and circular waveguide irises. Figs. 9 and 10 represent the comparison of the dispersion and mean coupling impedance between elliptical and circular irises, respectively. In one case, the circular waveguide iris is inscribed to the elliptical iris, and in the second case the circular waveguide iris circumscribes the elliptical iris. The layout and dimensions of the periodic structures under analysis are shown in the caption of Fig. 9.

From these dispersion curves, it can be seen that if the section of the elliptical waveguide iris is an inscribed circular waveguide, then the dispersion will increase in comparison with the circular structure; if the section is a circumscribed one, then the dispersion will decrease. However, from Fig. 10, we conclude that using elliptical waveguide iris will strongly strengthen the coupling impedance. In conclusion, we can achieve a high value of the interaction between an RF wave and the electron beam only using elliptical waveguide irises in corrugated cylindrical waveguides.

Fig. 11 shows the normalized longitudinal electric field representation plotted in the transverse plane for the first $T M$ 


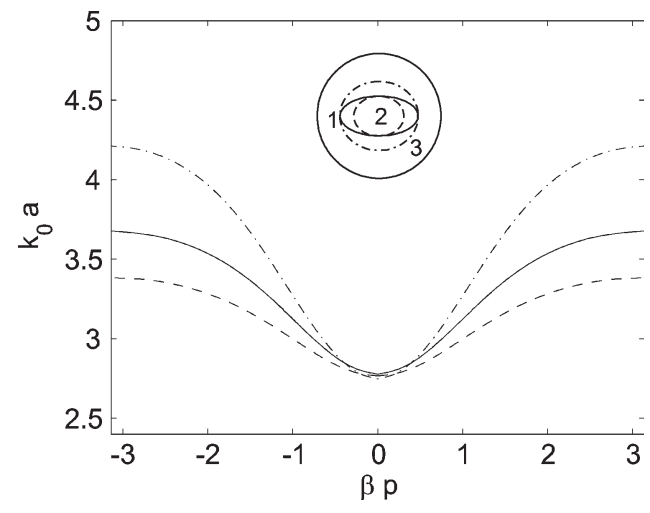

Fig. 9. Brillouin diagram for a periodic circular structure loaded with elliptical waveguide irises (solid line) and two different circular irises (dashed and dash-dotted lines). Dimensions of the structures: period $p=2 \mathrm{~mm}$ and $d=1 \mathrm{~mm}$; circular bigger radius $a=5 \mathrm{~mm}$; elliptical iris dimensions $a_{1}=3.5 \mathrm{~mm}, b_{1}=2.87 \mathrm{~mm}$; and circular iris radius $b=2.87 \mathrm{~mm}$ (circular iris inscribed of the elliptical iris case, represented with dashed line) and $b=3.5 \mathrm{~mm}$ (circular iris circumscribed of the elliptical case, represented with dash-dotted line), respectively.

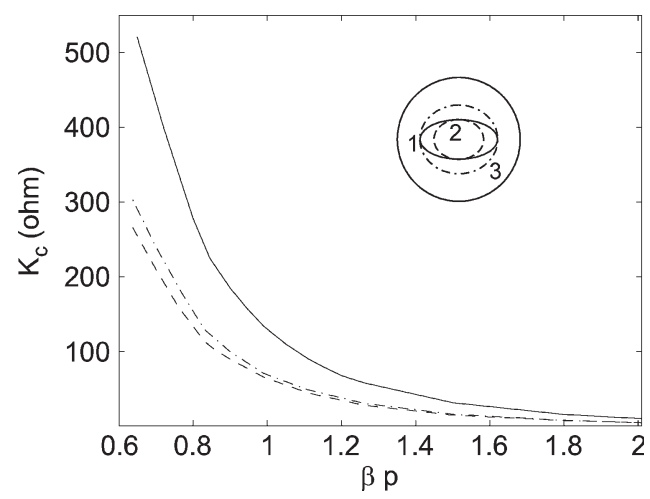

Fig. 10. Mean coupling impedance for the same periodic structures in Fig. 9. (Solid line) Elliptical iris case. (Dashed line) Inscribed circular case. (Dashdotted line) Circumscribed circular case.

mode $\left({ }_{e} T M_{01}\right)$, for the fourth $T M$ mode $\left({ }_{e} T M_{21}\right)$, and for the sixth $T M$ mode $\left({ }_{e} T M_{02}\right)$ of the elliptical waveguide iris studied in Fig. 9 and computed at 40, 80, and $90 \mathrm{GHz}$, respectively. These three modes have cutoff frequencies of $f_{c}=36.542 \mathrm{GHz}, f_{c}=74.523 \mathrm{GHz}$, and $f_{c}=87.015 \mathrm{GHz}$, respectively. The cutoff frequency as well as the electric field has been obtained by means of the BI-RME method, enclosing the elliptical waveguide iris in a square waveguide of size $10 \mathrm{~mm}$.

Finally, to demonstrate the efficiency of the method proposed, the Brioullin diagram of the first five Floquet modes (symmetric and non-symmetric modes such as the $T M_{01}$, $T E_{21}, T M_{21}, T E_{41}$, and $T M_{02}$ of a circular waveguide or first $5 T M / T E_{2 m n}$ modes) for the same periodic circular structure loaded with elliptical waveguide irises are presented in Fig. 12. The fundamental mode is represented in solid line; this Floquet mode propagates for $1.1 \leq k_{0} p \leq 1.48$ and for $k_{0} p \geq 3.5$ in the considered frequency band. The second Floquet mode of a circular waveguide, in dashed lines, propagates for $1.88 \leq$ $k_{0} p \leq 1.99$, for $2.82 \leq k_{0} p \leq 2.98$, and for $k_{0} p \geq 3.22$. The third Floquet mode is represented in dash-dotted line, and the fourth Floquet mode is represented in dotted lines, respectively.

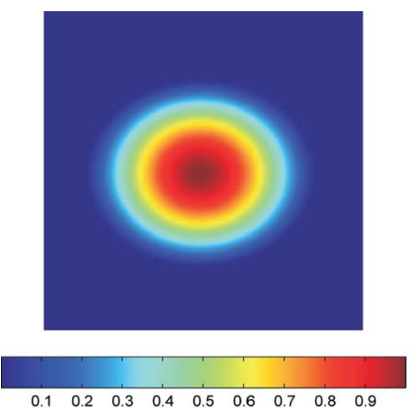

(a)

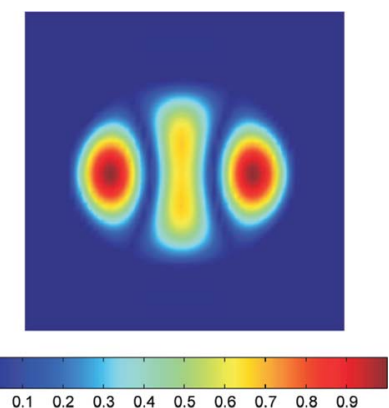

(b)

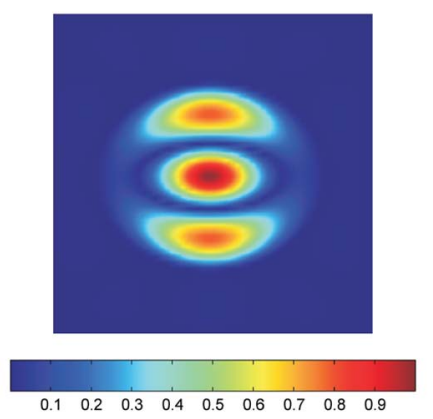

(c)

Fig. 11. Normalized longitudinal electric field representation plotted in the transverse plane (a) for the first $T M$ mode $\left(e T M_{01}\right)$, (b) for the fourth TM mode $\left(e T M_{21}\right)$, and (c) for the sixth TM mode $\left(e T M_{02}\right)$ of the elliptical waveguide iris studied in Fig. 9.

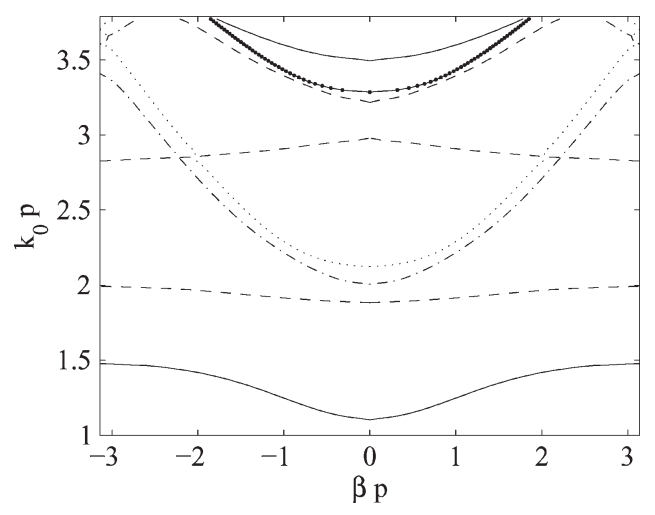

Fig. 12. Brillouin diagram for a periodic circular structure loaded with elliptical waveguide irises. Dimensions of the structure: circular radius $a=5 \mathrm{~mm}$, elliptical iris dimensions $a_{1}=3.5 \mathrm{~mm}, b_{1}=2.87 \mathrm{~mm}$, period $p=2 \mathrm{~mm}$, and $d=1 \mathrm{~mm}$. In the figure, the different lines represent the first five TM/TE Floquet modes propagating in the structure (such as the $T M_{01}, T E_{21}, T M_{21}$, $T E_{41}$, and $T M_{02}$ of a circular waveguide or $T M / T E_{2 m n}$ modes).

The second symmetric mode appears from $k_{0} p \geq 3.28$, and it is represented with a solid-point line. An intensive use of the proposed sorting technique based on the correlation matrix has been required to find the correct solution, since the first five Floquet modes of (7) are mixed up in the considered frequency band (from 24 to $90 \mathrm{GHz}$ ). Such results have been obtained using 150 accessible modes, 200 basis functions, and 500 kernel terms (i.e., localized modes [19]) for solving the planar waveguide junctions of the structure. To compute the displayed dispersion diagram, the first six $T M / T E_{2 m n}$ accessible modes of the periodic structure have been used for solving (8). The complete analysis procedure has required a global computational effort of $0.76 \mathrm{~s}$ per frequency point. 


\section{CONCLUSION}

An efficient tool for the full-wave modal characterization of periodic structures loaded with elliptical waveguides has been described. This tool allows the accurate computation of the dispersion diagram, which is related to the lowest symmetric mode and also to nonsymmetric and higher-order Floquet modes, of the cited periodic structures. This novel analysis tool is based on the combination of an integral equation technique and the wellknown BI-RME method, thus leading to the final solution of a standard eigenvalue matrix problem. For validation purposes, such method has first been applied to geometries already considered in the technical literature. Then, the dispersion diagrams and the interaction impedance of asymmetric periodic elliptical waveguides as well as periodic circular waveguide with elliptical irises have been studied. From the computed results, we can state that decreasing the minor axis length in the waveguide structure or/and in the iris increases the interaction impedance while the dispersion strengthens, in comparison with the circular case. The lengthening of the major axis can improve the dispersion bandwidth. Moreover, for high frequencies, the coupling impedance decreases very slowly. Periodic structures loaded with elliptical waveguides have potential applications to high-power microwave devices and filtering networks at millimeter-wave frequencies.

\section{REFERENCES}

[1] R. Barker and E. Schamiloglu, High-Power-Microwave Sources and Technologies. New York: IEEE Press, 2001, ser. IEEE Press Series on RF and Microwave Technology.

[2] W. Main, Y. Carmel, K. Ogura, J. Weaver, G. Nusinovich, S. Kobayashi, J. P. Tate, J. Rodgers, A. Bromborsky, S. Watanabe, M. R. Amin, K. Minami, W. W. Destler, and V. L. Granatstein, "Electromagnetic properties of open and closed overmoded slow-wave resonators for interaction with relativistic electron beams," IEEE Trans. Plasma Sci., vol. 22, no. 5, pp. 566-577, Oct. 1994.

[3] F. Kantrowitz and E. Adler, "Calculation of $T M_{0 n}$ mode dispersion relations in rippled-wall waveguides," IEEE Trans. Electron Devices, vol. 37, no. 12, pp. 2619-2621, Dec. 1990.

[4] S. Bugaev, V. Cherepenin, V. Kanavets, A. Klimov, A. Kopenkin, V. Koshelev, V. Popov, and A. Slepkov, "Relativistic multiwave Cherenkov generators," IEEE Trans. Plasma Sci., vol. 18, no. 3, pp. 525536, Jun. 1990.

[5] A. Bromborsky and B. Ruth, "Calculation of $T M_{0 n}$ mode dispersion relations in a corrugated cylindrical waveguide," IEEE Trans. Microw. Theory Tech., vol. MTT-32, no. 6, pp. 600-605, Jun. 1984.

[6] H. Guo, Y. Carmel, W. Lou, L. Chen, J. Rodgers, D. Abe, A. Bromborsky, W. Destler, and V. Granatstein, "A novel highly accurate synthetic technique for determination of the dispersive characteristics in periodic slow wave circuits," IEEE Trans. Microw. Theory Tech., vol. 40, no. 11, pp. 2086-2094, Nov. 1992.

[7] H. Wang, Z. Yang, L. Zhao, and Z. Liang, "Numerical computation of dispersion curves for symmetric and asymmetric modes in arbitrary cylindrical metal SWS," IEEE Trans. Plasma Sci., vol. 33, no. 1, pp. 111-118, Feb. 2005.

[8] K. Tanaka, K. Minami, X. Zheng, Y. Carmel, A. Vlasov, and V. Granatstein, "Propagating quasi-TE modes in a vacuum axisymmetric corrugated-wall waveguide," IEEE Trans. Plasma Sci., vol. 26, no. 3, pp. 940-946, Jun. 1998.

[9] P. Wang, X. Zhou, J. A. Nation, S. Banna, and L. Schachter, "Symmetric and asymmetric mode interaction in high-power traveling wave amplifiers: Experiments and theory," IEEE Trans. Plasma Sci., vol. 28, no. 6, pp. 2262-2271, Dec. 2000.

[10] A. N. Vlasov, A. G. Shkvarunets, J. C. Rodgers, Y. Carmel, T. M. Antonsen, T. M. Abuelfadl, D. Lingze, V. A. Cherepenin, G. S. Nusinovich, M. Botton, and V. Granatstein, "Overmoded GW-class surface wave microwave oscillator," IEEE Trans. Plasma Sci., vol. 28, no. 3, pp. 550-560, Jun. 2000
[11] R. Bhatt, T. Bemis, and C. Chen, "Three-dimensional theory and simulation of non relativistic elliptic electron and ion beam generation," IEEE Trans. Plasma Sci., vol. 34, no. 2, pp. 187-193, Apr. 2006.

[12] W. Wang, Y. Wei, and Y. Gong, "Review of the novel slow-wave structures for high-power traveling-wave tube," Int. J. Infrared Millim. Waves, vol. 24, no. 9, pp. 1469-1484, Sep. 2003.

[13] P. J. B. Clarricoats and A. D. Olver, Corrugated Horns for Microwave Antennas. Stevenage, U.K.: Peregrinus, 1984.

[14] S. B. Raevskii and V. I. Smorgonskii, "Dispersion equation of corrugated elliptical waveguide," Radio Eng. Electron. Phys., vol. 17, no. 6, pp. 1297-1300, Jun. 1972.

[15] I. G. Belov, V. A. Kalmyk, S. B. Raevskii, and V. I. Smorgonskii, "Computation of critical frequencies and dispersion characteristics of corrugated elliptical waveguide," Radio Eng. Electron. Phys., vol. 18, no. 10, pp. 1591-1593, Oct. 1973.

[16] J. Xu, W. Wang, L. Yue, Y. Wei, and Y. Gong, "Study of corrugated elliptical waveguides for slow-wave structures," IEEE Trans. Electron Devices, vol. 54, no. 1, pp. 151-156, Jan. 2007.

[17] J. Xu, W. Wang, L. Yue, Y. Wei, and Y. Gong, "Slow-wave characteristics of elliptical corrugated waveguides with a concentric circular hole," Chin. Phys. Lett., vol. 23, no. 1, pp. 243-246, Jan. 2006.

[18] T. C. Han, F. Y. Xu, and G. J. Li, "Transmission characteristics of corrugated elliptic guide considering higher order modes in the slot region," Prog. Electromagn. Res. Lett., vol. 3, pp. 79-85, 2008.

[19] G. Gerini, M. Guglielmi, and G. Lastoria, "Efficient integral equation formulations for admittance or impedance representation of planar waveguide junctions," in Proc. IEEE MTT-S Int. Microw. Symp. Dig., Jun. 1998, vol. 3, pp. 1747-1750.

[20] G. Conciauro, M. Bressan, and C. Zuffada, "Waveguide modes via an integral equation leading to a linear matrix eigenvalue problem," IEEE Trans. Microw. Theory Tech., vol. MTT-32, no. 11, pp. 1495-1504, Nov. 1984.

[21] S. Cogollos, S. Marini, V. E. Boria, P. Soto, A. Vidal, H. Esteban, J. V. Morro, and B. Gimeno, "Efficient modal analysis of arbitrarily shaped waveguides composed of linear, circular and elliptical arcs using the BIRME method," IEEE Trans. Microw. Theory Tech., vol. 51, no. 12, pp. 2378-2390, Dec. 2003.

[22] R. E. Collin, Field Theory of Guided Waves. New York: McGraw-Hill, 1960.

[23] M. Bozzi, L. Germani, S. Minelli, L. Perregrini, and P. de Maagt, "Efficient calculation of the dispersion diagram of planar electromagnetic band-gap structures by the MoM/BIRME method," IEEE Trans. Antennas Propag., vol. 53, no. 1, pp. 29-34, Jan. 2005.

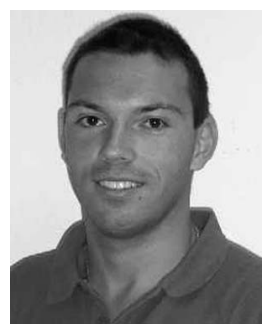

Stephan Marini was born in Cagli, Italy, on January 3, 1976. He received the Laurea degree in electronics engineering from the University of Perugia, Perugia, Italy, in 2001 and the Ph.D. degree in telecommunications from the Universidad Politécnica de Valencia, Valencia, Spain, in 2005.

In June 2001, he was with the Departamento de Comunicaciones, Universidad Politécnica de Valencia, in the frame of the European Union project "Millimeter-wave and Microwave Components Design Framework for Ground and Space Multimedia Network." Since 2005, he has been a Lecturer with the Departamento de Física, Ingeniería de Sistemas y Teoría de la Señal, Universidad de Alicante, Alicante, Spain. In 2009, he obtained the "José Castillejo" postdoctoral fellowship from the Spanish Government for a short stay (four months) with the LEMA Laboratory, École Polytechnique Fédérale de Lausanne, Lausanne, Switzerland. His research interests include numerical methods for the analysis of arbitraryshaped waveguide, scattering, and periodic structures. 


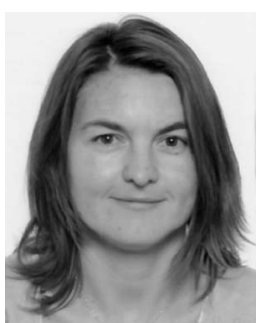

Ángela Coves (M'04) was born in Alicante, Spain, on May 20, 1976. She received the Licenciado degree in physics and the Ph.D. degree from the Universidad de Valencia, Valencia, Spain, in 1999 and 2004, respectively.

Since 2001, she has been a Lecturer with the Signal Theory and Communications Division, Universidad Miguel Hernández (UMH), Alicante. Her current research interests include the study of electron discharges in microwave components and computer-aided techniques of the analysis of microwave passive components, waveguide structures, and diffraction gratings.

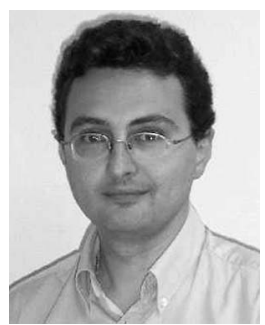

Vicente E. Boria (S'91-A'99-SM'02) was born in Valencia, Spain, on May 18, 1970. He received the "Ingeniero de Telecomunicación" degree (with first-class honors) and the "Doctor Ingeniero de Telecomunicación" degree from the Universidad Politécnica de Valencia, Valencia, in 1993 and 1997, respectively.

In 1993, he joined the "Departamento de Comunicaciones," Universidad Politécnica de Valencia, where he has been a Full Professor since 2003. In 1995 and 1996, he was holding a Spanish Trainee position with the European Space Research and Technology Centre (ESTEC)European Space Agency (ESA), Noordwijk, The Netherlands, where he was involved in the area of EM analysis and design of passive waveguide devices. He has authored or coauthored five chapters in technical textbooks, 65 papers in refereed international technical journals, and over 150 papers in international conference proceedings. His current research interests are focused on the analysis and automated design of passive components, left-handed and periodic structures, as well as on the simulation and measurement of power effects (multipactor and corona) in passive waveguide systems.

Dr. Boria has been a member of the IEEE Microwave Theory and Techniques Society (IEEE MTT-S) and the IEEE Antennas and Propagation Society (IEEE AP-S) since 1992. He is a member of the Editorial Boards of the IEEE TRANSACTIONS ON MicrowaVe THEORY AND TECHNIQUES and the IEEE Microwave AND Wireless COMPONENTS LETTERS. He is also a member of the Technical Committees of the IEEE-MTT International Microwave Symposium and of the European Microwave Conference. He was the recipient of the 2001 Social Council of Universidad Politécnica de Valencia First Research Prize for his outstanding activity during the period 1995-2000.

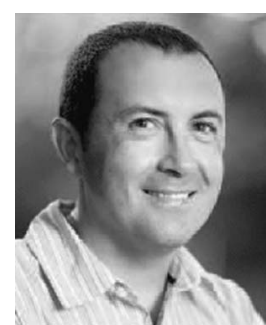

Benito Gimeno (M’01) was born in Valencia, Spain, on January 29, 1964. He received the Licenciado degree in physics and the Ph.D. degree from the Universidad de Valencia, Valencia, in 1987 and 1992, respectively.

From 1987 to 1990, he was a Fellow with the Universidad de Valencia. Since 1990, he has been an Assistant Professor with the Departamento de Física Aplicada y Electromagnetismo, Universidad de Valencia, where he became an Associate Professor in 1997. He was working with the European Space Research and Technology Centre of the European Space Agency (ESTEC) as a Research Fellow during 1994 and 1995. In 2003, he obtained a fellowship from the Spanish Government for a short stay (three months) as a Visiting Scientific with the Università degli Studi di Pavia, Pavia, Italy. His current research interests include the areas of computer-aided techniques for the analysis of passive components for space applications, waveguides, and cavities including dielectric objects, electromagnetic band-gap structures, frequency selective surfaces, and nonlinear phenomena appearing in power microwave subsystems (multipactor and corona effects). 